\title{
A NEW FINITE CONTINUATION ALGORITHM FOR LINEAR PROGRAMMING*
}

\author{
KAJ MADSEN ${ }^{\dagger}$, HANS BRUUN NIELSEN ${ }^{\ddagger}$, AND MUSTAFA Ç. PINAR $\ddagger$
}

\begin{abstract}
We describe a new finite continuation algorithm for linear programming. The dual of the linear programming problem with unit lower and upper bounds is formulated as an $\ell_{1}$ minimization problem augmented with the addition of a linear term. This nondifferentiable problem is approximated by a smooth problem. It is shown that the minimizers of the smooth problem define a family of piecewise-linear paths as a function of a smoothing parameter. Based on this property, a finite algorithm that traces these paths to arrive at an optimal solution of the linear program is developed. The smooth problems are solved by a Newton-type algorithm. Preliminary numerical results indicate that the new algorithm is promising.
\end{abstract}

Key words. finite algorithms, continuation methods, linear programming, $\ell_{1}$ optimization, Huber function, Newton's method

AMS subject classifications. 90C05, 65K05

1. Introduction. This paper introduces a new finite algorithm for linear programming (LP) by taking an unconstrained view of the problem. The algorithm operates on a special form of the linear program. We consider an LP problem where the variables are restricted to be between -1 and +1 . We call this problem a "normalized" linear program. It is well known $[3,14]$ that the dual of this linear program can be formulated as an unconstrained "augmented" $\ell_{1}$ minimization problem. The term "augmented" is used in this context to indicate that the function to be minimized is composed of an $\ell_{1}$ term and a linear term. In this paper, the $\ell_{1}$ norm is approximated by a smooth "Huber" function [10]. The approximation involves a positive smoothing parameter $\gamma$. We also refer to this smooth problem as the "Huber" problem. The following properties of the approximation are emphasized as the main contributions of our analysis in this paper.

(P0) The minimizers of the smooth problem define a family of piecewise-linear paths as a function of the smoothing parameter $\gamma$ (Lemma 3 ).

(P1) The solution to the LP problem is detected for sufficiently small $\gamma>0$ using these paths. That is, $\gamma$ does not have to be decreased to zero in order to obtain an exact solution to the LP problem (Theorem 1).

(P2) The structure of the set of minimizers of the smooth problem closely "mimics" the structure of the solution set of the $\ell_{1}$ and LP problems (Theorems 1 and 4).

(P3) The nature of the approximation allows a constructive duality link to the original primal problem, thereby giving the resulting algorithm a primal-dual flavor (Theorem 4).

These results generalize the results of an earlier study on the $\ell_{1}$ problem by the first two authors [13].

These properties suggest an algorithm to trace these piecewise-linear paths to arrive at a solution of the linear program. We refer to these paths as "solution paths" throughout the paper. We note that the solution path could be unique as is the case

* Received by the editors November 16, 1993; accepted for publication (in revised form) December 28,1994 . This research was partially supported by Danish National Science Council grant 11-0505.

$\dagger$ Institute of Mathematical Modelling, Numerical Analysis Group, Technical University of Denmark, 2800 Lyngby, Denmark (km@imm.dtu.dk).

$\ddagger$ Department of Industrial Engineering, Bilkent University, 06533 Bilkent, Ankara, Turkey. 
with the concept of the central path in interior point methods. Our algorithm is best interpreted as a continuation algorithm since it possesses the following main features of continuation algorithms.

1. The solution of a parametrized family of subproblems as a parameter varies over an interval-in our case the smooth "Huber" problem as a function of the smoothing parameter $\gamma$.

2. The use of information from the solutions of the previous problems to aid in the solution of the next member of the family. Here, we utilize the piecewiselinear dependence of the solution paths on $\gamma$ and the duality gap to compute an initial point for subsequent Huber problems. Using information from the previous solution makes the convergence of subsequent iterations faster.

3. A method for adjusting the continuation parameter for the efficient and accurate solution of subsequent problems. Piecewise linearity of the solution paths is again exploited in devising an efficient scheme for adjusting $\gamma$.

4. The use of a local iterative method to solve the subproblems. We use a finite Newton method [12] to solve the smooth Huber problem.

As a result of $(\mathrm{P} 1)$, the continuation algorithm is a finite procedure provided that $\gamma$ is decreased by at least a certain factor after each unconstrained minimization. We discuss these ideas more precisely in the forthcoming sections.

Our algorithm is related to many ideas in the optimization literature. In particular, there exists a close relationship between our method and penalty function algorithms since these methods can also be interpreted as continuation methods. Penalty methods usually treat all or a subset of the constraints of an optimization problem appending a penalty term to the objective function. The resulting penalty problem is solved by an iterative method, and solutions of the previous problems are used as starting points for the solution of subsequent problems. The process is controlled using a penalty parameter that is adjusted after the solution of each penalty problem if certain optimality criteria are not met. Barrier function and interior point algorithms can also be subsumed under this category, as well as more recent noninterior continuation algorithms [2]. In this regard our algorithm offers a novelty in the utilization of piecewise linearity and the absence of a penalty function.

The use of the $\ell_{1}$ function in LP was proposed by Conn in [5] and later studied by Bartels in [1]. In these studies the $\ell_{1}$ function is used as a penalty function. The resulting unconstrained piecewise-linear problem is solved using a combined projected descent and active set strategy.

Another related idea is due to Pinar and Zenios [19], where the Huber function is utilized as a linear-quadratic penalty function to penalize the side constraints in network models with side constraints and multicommodity network models. Linearquadratic functions have also been the subject of study by Rockafellar [20] and Sun [21].

A more recent idea was proposed by Coleman and $\mathrm{Li}$ in $[4,3]$. Their departure point is identical to ours in that they also work on the augmented $\ell_{1}$ dual of a normalized linear program. They develop an affine scaling algorithm to solve the augmented $\ell_{1}$ problem after a slight reformulation. Unfortunately, an implementation of their algorithm is not available for comparison.

To the best of our knowledge, our algorithm stands as a novel contribution to the linear optimization literature while it displays similarities with its chief competitors. In particular, the main computational burden is the solution of symmetric positive (semi)definite systems of linear equations reminiscent of interior point methods. On 
the other hand, two successive systems are closely related enough to warrant an update of the previous factorization, much like the simplex method. However, the iterates are neither required to stay in the interior nor on the surface of the polytope. Furthermore, the new algorithm stops when the duality gap is closed.

We develop a preliminary, but stable and efficient, implementation of the new algorithm for problems with full matrices. The algorithm is tested both on randomly generated problems and some problems from the Netlib collection. The results indicate a modest growth in the number of iterations as the problem size increases. We compare our results with a dense simplex subroutine from the NAG subroutine library based on the package LSSOL of Stanford Systems Optimization Library. The algorithm outperforms the dense simplex subroutine by a wide margin on the randomly generated problems. It is also competitive with the simplex subroutine on most of the Netlib problems tried. We also offer some comparisons with interior point methods and in particular with the CPLEX ${ }^{\mathrm{TM}}$ Barrier package which implements the primaldual interior point algorithm [11]. Several issues that remain unresolved about the algorithm will be addressed in future studies, including the development of a sparse implementation.

The rest of the paper is organized as follows. After some preliminaries, we study the structure of the smooth Huber problem in $\S 2$. The connection between the $\ell_{1}$ and LP problems and the smooth Huber problem is studied in $\S 3$. The results of these two sections form a constructive theory in that they.suggest an algorithm. The algorithm is described in $\S 4$. Numerical results along with some implementation details are given in $\S 5$.

1.1. Preliminaries. We wish to solve the following problem:

[NormLP]

$$
\begin{array}{cr}
\underset{y}{\operatorname{maximize}} & c^{T} y \\
\text { subject to } & A y=b, \\
& -e \leq y \leq e
\end{array}
$$

where $c \in \Re^{m}, A \in \Re^{n \times m}, b \in \Re^{n}$, and $e=(1, \ldots, 1)$. More general problems can be transformed into [NormLP] if the bounds on $y$ can be replaced by general upper and lower bounds. Furthermore, as we see in $\S 5$, we will also be able to solve the general LP problem via [NormLP] using the idea of slack of variables and some artificial bounds. Therefore, we limit our study to [NormLP].

The new method is based on solving the dual problem to [NormLP]:

[AL1]

$$
\operatorname{minimize} G(x) \equiv\left\|A^{T} x-c\right\|_{1}+b^{T} x .
$$

A proof of the duality correspondence can be found, for instance, in [14]. If $x^{*}$ and $y^{*}$ are dual solutions then

$$
\begin{aligned}
& a_{i}^{T} x^{*}-c_{i}<0 \Rightarrow y_{i}^{*}=1, \\
& a_{i}^{T} x^{*}-c_{i}>0 \Rightarrow y_{i}^{*}=-1,
\end{aligned}
$$

where $a_{i}$ is the $i$ th column of $A$. Therefore, 
where the last equality is the duality equality and expresses the necessary condition for a solution of $[\mathrm{AL} 1]$ :

$$
\begin{array}{r}
-A y^{*}+b=0, \\
-e \leq y^{*} \leq e .
\end{array}
$$

The method for solving [AL1] is similar to that in [13] for minimizing the linear $\ell_{1}$ function. We estimate a minimizer of $G$ by solving a sequence of approximating smooth problems, each of which depends on a parameter $\gamma>0$. These problems are defined as follows. Let

$$
r(x)=A^{T} x-c
$$

and for a given threshold $\gamma>0$ define

$$
\mathbf{s}_{\gamma}(x)=\left[s_{\gamma 1}(x), \ldots, s_{\gamma m}(x)\right]
$$

with

$$
s_{\gamma i}(x)= \begin{cases}-1 & \text { if } r_{i}(x)<-\gamma \\ 0 & \text { if }\left|r_{i}(x)\right| \leq \gamma \\ 1 & \text { if } r_{i}(x)>\gamma\end{cases}
$$

$\mathbf{s}_{\gamma}(x)$ is a sign vector, and in general a sign vector is any vector $\mathbf{s} \in \Re^{m}$ with components $s_{i} \in\{-1,1,0\}$. For any sign vector $\mathbf{s}$ we define

$$
W_{\mathbf{s}}=\operatorname{diag}\left(w_{1}, \ldots, w_{m}\right),
$$

where

$$
w_{i}=1-s_{i}^{2} .
$$

If $\mathbf{s}=\mathbf{s}_{\gamma}(x)$ then we also denote $W_{\mathbf{s}}$ by $W_{\gamma}(x)$ or $W_{\gamma}$ if no confusion is possible.

Now, the nondifferentiable problem [AL1] is approximated by the smooth "Huber problem" [10]:

[SAL1]

$$
\operatorname{minimize} G_{\gamma}(x) \equiv \frac{1}{2 \gamma} r^{T} W_{\gamma} r+\mathbf{s}_{\gamma}^{T}\left[r-\frac{1}{2} \gamma \mathbf{s}_{\gamma}\right]+b^{T} x
$$

where the argument $x$ is dropped for notational convenience. Clearly, $G_{\gamma}$ measures the "small" residuals $\left(\left|r_{i}(x)\right| \leq \gamma\right)$ by their squares while the "large" residuals are measured by the $\ell_{1}$ function. Thus, $G_{\gamma}$ is a piecewise quadratic function, and it is continuously differentiable in $\Re^{n}$. We prove that when $\gamma \rightarrow 0_{+}$any solution of [SAL1] is close to a solution of [AL1]. Furthermore, we show that dual solutions to [AL1] and [NormLP] can be detected directly when $\gamma$ is below a certain (problem dependent) threshold $\gamma_{0}>0$. Therefore, the new algorithm consists of solving [SAL1] for a decreasing set of threshold values $\gamma$ until dual solutions are detected. We summarize the algorithm as follows.

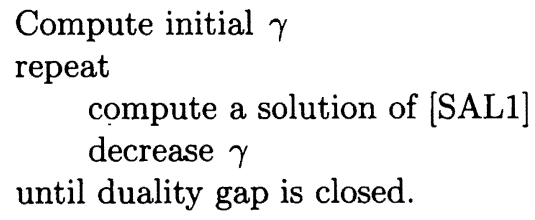

Since solving [SAL1] $\gamma>0$ is a finite procedure [12], the whole algorithm is finite provided $\gamma$ is decreased at least by a certain factor in each iteration. 
2. Properties of $G_{\gamma}$. In this section we describe some essential properties of $G_{\gamma}$.

We can assume without loss of generality that $A$ has rank $n$ and that every column $a_{i}$ of $A$ is nonzero. Otherwise, the problem could easily be reformulated to have these properties.

Clearly $G_{\gamma}$ is composed of a finite number of quadratic functions. In each domain $D \subseteq \Re^{n}$, where $\mathbf{s}_{\gamma}(x)$ is constant and $G_{\gamma}$ is equal to a specific quadratic function as seen from the above definition. These domains are separated by the following union of hyperplanes:

$$
B_{\gamma}=\left\{x \in \Re^{n}|\exists i:| r_{i}(x) \mid=\gamma\right\} .
$$

A sign vector $\mathbf{s}$ is $\gamma$-feasible at $x$ if

$$
\forall \varepsilon>0 \exists z \in \Re^{n} \backslash B_{\gamma}:\|x-z\|<\varepsilon \wedge \mathbf{s}=\mathbf{s}_{\gamma}(z) .
$$

If $\mathbf{s}$ is a $\gamma$-feasible sign vector at some point $x$, then let $Q_{\mathbf{s}}^{\gamma}$ be the quadratic function which equals $G_{\gamma}$ on the subset

$$
\mathcal{C}_{\mathbf{s}}^{\gamma}=\operatorname{cl}\left\{z \in \Re^{n} \mid \mathbf{s}_{\gamma}(z)=\mathbf{s}\right\} .
$$

$\mathcal{C}_{\mathbf{s}}^{\gamma}$ is called a $Q$-subset of $\Re^{n}$. Notice that any $x \in \Re^{n} \backslash B_{\gamma}$ has exactly one corresponding $Q$-subset $\left(\mathbf{s}=\mathbf{s}_{\gamma}(x)\right)$, whereas a point $x \in B_{\gamma}$ belongs to two or more $Q$-subsets. Therefore, in general, we must give a sign vector $\mathbf{s}$ in addition to $x$ in order to specify which quadratic function we are currently considering as representative of $G_{\gamma}$. However, the gradient of $G_{\gamma}$ is independent of the choice of s.

$Q_{\mathbf{s}}$ can be defined as follows:

$$
Q_{\mathbf{s}}(z)=\frac{1}{2 \gamma}(z-x)^{T}\left(A W_{\mathbf{s}} A^{T}\right)(z-x)+G_{\gamma}^{\prime T}(x)(z-x)+G_{\gamma}(x) .
$$

The gradient of the function $G_{\gamma}$ is given by

$$
G_{\gamma}^{\prime}(x)=A\left[\frac{1}{\gamma} W_{\mathbf{s}} r+\mathbf{s}\right]+b,
$$

where $\mathbf{s}$ is a $\gamma$-feasible sign vector at $x$. For $x \in \Re^{n} \backslash B_{\gamma}$, the Hessian of $G_{\gamma}$ exists and is given by

$$
G_{\gamma}^{\prime \prime}(x)=\frac{1}{\gamma} A W_{\gamma} A^{T} .
$$

The set of indices corresponding to "small" residuals

$$
\mathcal{A}_{\gamma}(z)=\left\{i \mid 1 \leq i \leq m \wedge s_{\gamma i}(z)=0\right\}
$$

is called the $\gamma$-active set at $z$ and the subspace

$$
\mathcal{V}_{\gamma}(z)=\operatorname{span}\left\{a_{i} \mid i \in \mathcal{A}_{\gamma}(z)\right\}
$$

is called the $\gamma$-active subspace at $z$. The set of minimizers of $G_{\gamma}$ is denoted by $M_{\gamma}$. In [12] it is shown that, for the case $b=\mathbf{0}$, there exists a minimizer $x_{\gamma} \in M_{\gamma}$ for which $\mathcal{V}_{\gamma}\left(x_{\gamma}\right)=\Re^{n}$. The idea of the proof is that if $\mathcal{V}_{\gamma}\left(z_{\gamma}\right) \neq \Re^{n}$ for a minimizer $z_{\gamma}$ then there exists a connected set of minimizers of the form $z_{\gamma}+\alpha h$, where $h \perp \mathcal{V}_{\gamma}\left(z_{\gamma}\right)$. 
By letting $\alpha$ increase (or decrease) from zero one can find another minimizer where the rank of the $\gamma$-active subspace is increased. Repeating this argument we finally find a minimizer where the rank of the corresponding $\gamma$-active subspace is maximal (i.e., the rank is equal to $n$ ). Clearly, this proof extends to the case $b \neq \mathbf{0}$.

Let $\mathbf{s}^{\gamma}(x)$ be the $\gamma$-feasible sign vector at $x$ with components

$$
s_{i}^{\gamma}(x)= \begin{cases}-1 & \text { if } r_{i}(x) \leq-\gamma \\ 0 & \text { if }\left|r_{i}(x)\right|<\gamma \\ 1 & \text { if } r_{i}(x) \geq \gamma\end{cases}
$$

Then we have the following.

LEMMA 1. $\mathbf{s}^{\gamma}(x)$ is constant for $x \in M_{\gamma}$. Furthermore, $r_{i}(x)$ is constant for $x \in M_{\gamma}$ if $s_{i}^{\gamma}=0$.

Proof. Let $z \in M_{\gamma}$ and let $\mathbf{s}=\mathbf{s}^{\gamma}(z)$; i.e., $G_{\gamma}(x)=Q_{\mathbf{s}}(x)$ for $x \in \mathcal{C}_{\mathbf{s}}^{\gamma}$. If $x \in \mathcal{C}_{\mathbf{s}}^{\gamma} \cap M_{\gamma}$ then $Q_{\mathbf{s}}^{\prime \prime}(x)(x-z)=0$. Therefore, if $\left|r_{i}(z)\right|<\gamma$ then $a_{i}^{T}(x-z)=0$ (see (16)), and hence $r_{i}(x)=r_{i}(z)$. Thus $r_{i}$ is constant in $\mathcal{C}_{\mathbf{s}}^{\gamma} \cap M_{\gamma}$. Using the fact that $M_{\gamma}$ is connected and $r_{i}$ is continuous, it is easily seen by repeating the argument above that $r_{i}$ is constant in $M_{\gamma}$. Next suppose $r_{i}(z) \geq \gamma$. Then $r_{i}(x) \geq \gamma$ for all $x \in M_{\gamma}$ because existence of $x \in M_{\gamma}$ with $r_{i}(x)<\gamma$ is excluded by the convexity of $M_{\gamma}$, the continuity of $r_{i}$, and the first part of the lemma. Similarly, $r_{i}(z) \leq-\gamma \Longrightarrow r_{i}(x) \leq-\gamma$ for $x \in M_{\gamma}$. This proves Lemma 1 .

Following the lemma it is meaningful to use the notation $\mathbf{s}^{\gamma}\left(M_{\gamma}\right)=\mathbf{s}^{\gamma}(x), x \in M_{\gamma}$ as the sign vector corresponding to the solution set. Lemma 1 has the following consequences which characterize the solution set $M_{\gamma}$.

CoROllary 1. $M_{\gamma}$ is a convex set that is contained in one $Q$-subset: $\mathcal{C}_{\mathbf{s}}^{\gamma}$ where $\mathbf{s}=\mathbf{s}^{\gamma}\left(M_{\gamma}\right)$.

Proof. The proof follows immediately from the linearity of the problem and Lemma 1.

CoROllaRY 2. Let $z \in M_{\gamma}$, and $\mathbf{s}=\mathbf{s}^{\gamma}\left(M_{\gamma}\right)$. Let $\mathcal{N}_{\mathbf{s}}$ be the orthogonal complement of $\mathcal{V}_{\mathbf{s}}=\operatorname{span}\left\{a_{i} \mid s_{i}=0\right\}$. Then

$$
M_{\gamma}=\left(z+\mathcal{N}_{\mathbf{s}}\right) \cap \mathcal{C}_{\mathbf{s}}^{\gamma}
$$

Proof. It follows from (17) that $G_{\gamma}^{\prime}(z+u)=0$ if $u \in \mathcal{N}_{\mathbf{s}}$ and $z+u \in \mathcal{C}_{\mathbf{s}}^{\gamma}$. Thus

$$
M_{\gamma} \supseteq\left(z+\mathcal{N}_{\mathbf{s}}\right) \cap \mathcal{C}_{\mathbf{s}}^{\gamma}
$$

If $x \in M_{\gamma}$ then $r_{i}(x)=r_{i}(z)$ for $s_{i}=0$, and hence $x-z \in \mathcal{N}_{\mathbf{s}}$. Therefore, Corollary 1 implies

$$
M_{\gamma} \subseteq\left(z+\mathcal{N}_{\mathbf{s}}\right) \cap \mathcal{C}_{\mathbf{s}}^{\gamma}
$$

which proves Corollary 2.

Notice that Corollary 2 has the following implication. Suppose the minimizer $z$ of $G_{\gamma}$ belongs to $B_{\gamma}$. Then $M_{\gamma}$ is the intersection of $\mathcal{C}_{\mathbf{s}}^{\gamma}$ with a space of dimension at least $p$ where $p=n-\operatorname{rank}\left\{a_{i}^{T}|| r_{i}(z) \mid<\gamma\right\}$. Therefore, one can normally expect multiple solutions if there exists a solution in $B_{\gamma}$.

3. The connection between $\boldsymbol{G}_{\gamma}, \boldsymbol{G}$, and [NormLP]. In this section we let the threshold $\gamma$ be variable and we analyze how the solution set $M_{\gamma}$ changes as $\gamma$ decreases and approaches zero. Furthermore, we show how to identify a solution to [NormLP]. 
Assume $x_{\gamma} \in M_{\gamma}$, and let $\mathbf{s}=\mathbf{s}^{\gamma}\left(M_{\gamma}\right)$. Let $\mathcal{V}_{\mathbf{s}}$ and $\mathcal{N}_{\mathbf{s}}$ be defined as in Corollary 2 (i.e., if $x_{\gamma} \notin B_{\gamma}$ then $\mathcal{V}_{\mathbf{s}}=\mathcal{V}_{\gamma}\left(x_{\gamma}\right)$ ).

Since $x_{\gamma}$ satisfies the necessary condition for a minimizer of $G_{\gamma}$, i.e.,

$$
\mathbf{0}=A W_{\mathbf{s}}\left(A^{T} x_{\gamma}-c\right)+\gamma(A \mathbf{s}+b)
$$

the following linear system is consistent:

$$
\left(A W_{\mathbf{s}} A^{T}\right) d=A \mathbf{s}+b
$$

Inserting (23) into (22) we see that $x_{0} \equiv x_{\gamma}+\gamma d$ is the least squares solution to the linear system

$$
W_{\mathbf{s}}\left(A^{T} z-c\right)=\mathbf{0}
$$

LeMma 2. Assume that the linear system (24) is consistent. Then

$$
\frac{1}{\gamma} W_{\mathbf{s}} r\left(x_{\gamma}\right)=-W_{\mathbf{s}} A^{T} d,
$$

where $d$ is a solution to (23).

Proof. The proof follows by inserting the solution $\left(x_{\gamma}+\gamma d\right)$ into (24).

Now let $d$ solve (23) and assume $\mathbf{s}^{\gamma-\varepsilon}\left(x_{\gamma}+\varepsilon d\right)=\mathbf{s}$; i.e., $x_{\gamma}+\varepsilon d \in \mathcal{C}_{\mathbf{s}}^{\gamma-\varepsilon}$ for some $\varepsilon>0$. The linearity of the problem implies $x_{\gamma}+\delta d \in \mathcal{C}_{\mathbf{s}}^{\gamma-\delta}$ for $0 \leq \delta \leq \varepsilon$. Therefore (22) and (23) show that $\left(x_{\gamma}+\delta d\right)$ is a minimizer of $G_{\gamma-\delta}$. Thus we have the following consequence of Corollary 2 .

LEMMA 3. Let $x_{\gamma} \in M_{\gamma}$ and let $\mathbf{s}=\mathbf{s}^{\gamma}\left(M_{\gamma}\right)$. Let $d$ solve (23). If $\mathbf{s}^{\gamma-\varepsilon}\left(x_{\gamma}+\right.$ $\varepsilon d)=\mathbf{s}$ for $\varepsilon>0$ then $\mathbf{s}^{\gamma-\delta}\left(x_{\gamma}+\delta d\right)=\mathbf{s}$, and

$$
M_{\gamma-\delta}=\left(x_{\gamma}+\delta d+\mathcal{N}_{\mathbf{s}}\right) \cap \mathcal{C}_{\mathbf{s}}^{\gamma-\delta}
$$

for $0 \leq \delta \leq \varepsilon$.

Lemma 3 states that the minimizers of $G_{\gamma}$ form a family of piecewise-linear paths as a function of the parameter $\gamma$.

THEOREM 1. There exists $\gamma_{0}>0$ such that $\mathbf{s}^{\gamma}\left(M_{\gamma}\right)$ is constant for $0<\gamma \leq \gamma_{0}$. Furthermore,

$$
M_{\gamma-\delta}=\left(x_{\gamma}+\delta d+\mathcal{N}_{\mathbf{s}}\right) \cap \mathcal{C}_{\mathbf{s}}^{\gamma-\delta} \text { for } 0 \leq \delta<\gamma \leq \gamma_{0},
$$

where $\mathbf{s}=s^{\gamma}\left(M_{\gamma}\right)$ and $d$ solves $(23)$.

Proof. Since there are only a finite number of different sign vectors the theorem is a consequence of Lemma 3.

Corollary 3. Let $0<\gamma \leq \gamma_{0}$ and $\mathbf{s}=\mathbf{s}^{\gamma}\left(M_{\gamma}\right)$. Let $x_{\gamma} \in M_{\gamma}$. If $s_{i}=0$ then

$$
r_{i}\left(x_{\gamma}\right)=-\left(a_{i}^{T} d\right) \gamma
$$

where $d$ is any solution of $(23)$; i.e., $r_{i}\left(x_{\gamma}\right) / \gamma$ is constant.

Proof. Let $s_{i}=0$. Since Theorem 1 implies $\left|r_{i}\left(x_{\gamma}+\delta d\right)\right|<\gamma-\delta$ for $0 \leq \delta<\gamma$ we obtain $r_{i}\left(x_{\gamma}+\gamma d\right)=0$. Therefore (24) is consistent and the result is a consequence of Lemma 2.

If $x_{\gamma} \in M_{\gamma}$ then $y_{\gamma}=-\left(W_{\mathbf{s}} r\left(x_{\gamma}\right) / \gamma+\mathbf{s}\right)$, where $\mathbf{s}=\mathbf{s}^{\gamma}\left(M_{\gamma}\right)$, is feasible in [NormLP] as it is seen from (22). $y_{\gamma}$ and $x_{\gamma}$ are called dual solutions, and we show that $y_{\gamma}$ solves [NormLP] when $\gamma$ is small enough. 
In the following two theorems we show that one can often expect the gap between the dual objective function $G$ and the primal objective $c^{T} y_{\gamma}$ to decrease from $x_{\gamma}$ in the direction $d$. In fact, we prove that the primal objective always increases and that $G$ decreases under a certain assumption. However, all our experience indicates that $G$ always decreases in the direction $d$ from $x_{\gamma}$, but we have not been able to prove it in general.

THEOREM 2. Let $x_{\gamma} \in M_{\gamma}$ and $\mathbf{s}=\mathbf{s}^{\gamma}\left(M_{\gamma}\right)$. Let $d$ solve (23) and let $x_{0}=$ $x_{\gamma}+\gamma d$. Let

$$
y_{\delta}=-\left(W_{\mathbf{s}} r\left(x_{0}-\delta d\right) / \delta+\mathbf{s}\right), \quad \delta>0
$$

and $H(\delta)=c^{T} y_{\delta}$. Then $H^{\prime}(\gamma) \leq 0$

Proof. Since $x_{0}$ is the least squares solution of (24), the vector

$$
\rho=W_{\mathbf{s}} A^{T} x_{0}-W_{\mathbf{s}} c
$$

is orthogonal to $W_{\mathbf{s}} A^{T} x_{0}$ and

$$
\left\|W_{\mathbf{s}} A^{T} x_{0}\right\|_{2}^{2}+\|\rho\|_{2}^{2}=\left\|W_{\mathbf{s}} c\right\|_{2}^{2}
$$

Therefore,

$$
\begin{aligned}
c^{T} W_{\mathbf{s}} r\left(x_{0}\right) & =c^{T} W_{\mathbf{s}} W_{\mathbf{s}} A^{T} x_{0}-c^{T} W_{\mathbf{s}} c \\
& =\left(W_{\mathbf{s}} A^{T} x_{0}-\rho\right)^{T}\left(W_{\mathbf{s}} A^{T} x_{0}\right)-\left\|W_{\mathbf{s}} c\right\|_{2}^{2} \\
& =-\|\rho\|_{2}^{2} \leq 0 .
\end{aligned}
$$

Furthermore,

$$
\begin{aligned}
H(\gamma) & =-c^{T}\left(W_{\mathbf{s}}\left(A^{T}\left(x_{0}-\gamma d\right)-c\right) / \gamma+\mathbf{s}\right) \\
& =-c^{T} W_{\mathbf{s}} r\left(x_{0}\right) / \gamma+c^{T} W_{\mathbf{s}} A^{T} d-c^{T} s
\end{aligned}
$$

Therefore (28) implies

$$
H^{\prime}(\gamma)=c^{T} W_{\mathbf{s}} r\left(x_{0}\right) / \gamma^{2} \leq 0
$$

It follows from the proof that if $(24)$ is consistent then $H^{\prime}(\gamma)=0$.

We now extend the definition of $\mathbf{s}_{\gamma}$ to the case $\gamma=0$ :

$$
s_{0 i}(x)= \begin{cases}-1 & \text { if } r_{i}(x)<0 \\ 0 & \text { if } r_{i}(x)=0 \\ 1 & \text { if } r_{i}(x)>0\end{cases}
$$

Furthermore, let

$$
\mathcal{C}_{\mathbf{s}}^{0}=\operatorname{cl}\left\{z \in \Re^{n} \mid \mathbf{s}_{0}(z)=\mathbf{s}\right\}
$$

THEOREM 3. Let $x_{\gamma} \in M_{\gamma}$. If (24) is consistent then the directional derivative

$$
\lim _{\delta \downarrow 0}\left\{\left(G\left(x_{\gamma}+\delta d\right)-G\left(x_{\gamma}\right)\right) / \delta\right\}
$$

is nonpositive. 
Proof. Let $\mathbf{s}=\mathbf{s}^{\gamma}\left(M_{\gamma}\right), \mathbf{s}_{0}=\mathbf{s}_{0}\left(x_{\gamma}\right)$, and $\mathcal{A}=\left\{i \mid s_{i}=0\right\}$. First we notice that for $i \in \mathcal{A}$ the consistency of (24) implies that if $r_{i}\left(x_{\gamma}\right)=0$ then $r_{i}\left(x_{\gamma}+\delta d\right)=0$ for any $\delta$. Therefore the following holds for $\delta$ being a sufficiently small positive number:

$$
\begin{aligned}
G\left(x_{\gamma}+\delta d\right) & =\mathbf{s}^{T} r\left(x_{\gamma}+\delta d\right)+\mathbf{s}_{0}^{T} W_{\mathbf{s}} r\left(x_{\gamma}+\delta d\right)+b^{T}\left(x_{\gamma}+\delta d\right) \\
& =G\left(x_{\gamma}\right)+\delta\left[\mathbf{s}^{T} A^{T} d+b^{T} d+\mathbf{s}_{0}^{T} W_{\mathbf{s}} A^{T} d\right] .
\end{aligned}
$$

Using (23) and (25) we obtain

$$
\begin{aligned}
{\left[G\left(x_{\gamma}+\delta d\right)-G\left(x_{\gamma}\right)\right] / \delta } & =d^{T} A W_{\mathbf{s}} A^{T} d+\mathbf{s}_{0}^{T} W_{\mathbf{s}} A^{T} d \\
& =d^{T} A W_{\mathbf{s}}^{T} W_{\mathbf{s}} A^{T} d+\mathbf{s}_{0}^{T} W_{\mathbf{s}} A^{T} d \\
& =\left(W_{\mathbf{s}} r\left(x_{\gamma}\right) / \gamma\right)^{T}\left(W_{\mathbf{s}} r\left(x_{\gamma}\right) / \gamma\right)-\mathbf{s}_{0}^{T} W_{\mathbf{s}} r\left(x_{\gamma}\right) / \gamma \\
& =\sum_{i \in \mathcal{A}}\left[\left(\frac{r_{i}\left(x_{\gamma}\right)}{\gamma}\right)^{2}-s_{0 i} \frac{r_{i}\left(x_{\gamma}\right)}{\gamma}\right] .
\end{aligned}
$$

The last sum is nonpositive because $\left|r_{i}\left(x_{\gamma}\right)\right|<\gamma$ for $i \in \mathcal{A}$. This proves the theorem.

THEOREM 4. Let $0<\gamma \leq \gamma_{0}$, where $\gamma_{0}$ is given in. Theorem 1 , and let $\mathbf{s}=$ $\mathbf{s}^{\gamma}\left(M_{\gamma}\right)$. Let $x_{\gamma} \in M_{\gamma}$ and $d$ solve (23). Then any point in the set

$$
M_{0}=\left(x_{\gamma}+\gamma d+\mathcal{N}_{\mathbf{s}}\right) \cap \mathcal{C}_{\mathbf{s}}^{0}
$$

solves [AL1], and

$$
y_{\gamma}=-\left(\frac{1}{\gamma} W_{\mathbf{s}} r\left(x_{\gamma}\right)+\mathbf{s}\right)
$$

solves [NormLP].

Proof. Assume $x_{0} \in M_{0}$. Then there exists a solution $d_{0}$ to (23) such that $x_{0}=x_{\gamma}+\gamma d_{0}$. Now the linearity and Theorem 1 imply that $x_{\gamma-\delta}=x_{\gamma}+\delta d_{0} \in M_{\gamma-\delta}$ for $0 \leq \delta \leq \gamma$.

Since $\mathbf{s}^{\gamma}\left(x_{\gamma}\right)=\mathbf{s}^{\gamma-\delta}\left(x_{\gamma-\delta}\right)$ for $0 \leq \delta<\gamma$, the continuity of $r$ gives

$$
r_{i}\left(x_{0}\right) \neq 0 \Longrightarrow \operatorname{sign}\left(r_{i}\left(x_{0}\right)\right)=\operatorname{sign}\left(r_{i}\left(x_{\gamma-\delta}\right)\right)=s_{i}=-y_{\gamma i}
$$

for $\delta$ close enough to $\gamma$. Therefore,

$$
\begin{aligned}
G\left(x_{0}\right) & =-r\left(x_{0}\right)^{T} y_{\gamma}+b^{T} x_{0} \\
& =-x_{0}^{T} A y_{\gamma}+c^{T} y_{\gamma}+x_{0}^{T} b \\
& =c^{T} y_{\gamma} .
\end{aligned}
$$

Furthermore, $y_{\gamma}$ is feasible for [NormLP]. Hence, $x_{0}$ and $y_{\gamma}$ are dual solutions to [AL1] and [NormLP]. Since this holds for any $x_{0} \in M_{0}$ the theorem is proved.

It is shown in [15] that $M_{0}$ coincides with the solution set of [AL1]. Notice that every $x_{0} \in M_{0}$ corresponds to the same dual solution $y_{\gamma}$. This means that if $M_{0}$ contains more than one point then the Lagrange multipliers corresponding to $y_{\gamma}$ are nonunique; i.e., $y_{\gamma}$ is a degenerate solution to [NormLP]. On the other hand, if [AL1] has a degenerate solution (i.e., nonunique Lagrange multiplier) then [NormLP] has multiple solutions. In that case, Theorem 4 picks one specific solution $y_{\gamma}$ to [NormLP]. 
4. The algorithm. The new algorithm is based on minimizing the function $G_{\gamma}$ for a set of decreasing values of $\gamma$. It can be described as follows. Starting from a point $x$, we find a minimizer of $G_{\gamma}$ for some $\gamma>0$. For example, we locate a path among the possibly infinitely many paths defined by the minimizers of $G_{\gamma}$. Utilizing the piecewise-linear dependence of the paths on $\gamma$ (Lemma 3) and the duality gap, we compute a guess at the minimizer of $G_{\gamma}$ for a smaller value of $\gamma$. Starting from this guess, we compute the exact minimizer of $G_{\gamma}$ using a Newton-type algorithm. Note that this gives the algorithm a predictor-corrector flavor in that we allow ourselves to deviate from the path only to return to it later from the predicted point using the Newton algorithm. Hence, we follow the solution paths closely without having to stay on any of them. Based on Theorem 4, this process terminates when the duality gap is closed.

To initiate the algorithm, a starting point $x^{0}$ and threshold value $\gamma^{0}$ are found as follows. Compute a least squares solution, i.e., a minimum $x_{l s}$ of the quadratic function $f(x) \equiv r^{T}(x) r(x)+b^{T} x$, by solving

$$
\left(A A^{T}\right) x_{l s}=A c-\frac{1}{2} b .
$$

Then set $x^{0}=x_{l s}$ and $\gamma^{0}$ such that $\left|\mathcal{A}_{\gamma^{0}}\left(x^{0}\right)\right| \geq n$.

The algorithm has two main components: (1) the solution of the smooth problem, i.e., minimization of $G_{\gamma}$ for a given value of $\gamma$, and (2) the reduction of $\gamma$ and the computation of an initial point for the solution of the subsequent Huber problem. We now consider these two components in detail.

4.1. Computing a minimizer of $G_{\gamma}$. The algorithm for computing a minimizer $x_{\gamma}$ of $G_{\gamma}$ is based on a modified Newton algorithm given in [12]. A search direction $h$ is computed by minimizing the quadratic $Q_{\mathbf{s}}$ where $\mathbf{s}=\mathbf{s}_{\gamma}(x)$ and $x$ is the current iterate. More precisely, we consider the equation

$$
Q_{\mathbf{s}}^{\prime \prime} h=-Q_{\mathbf{s}}^{\prime}(x),
$$

where $Q_{\mathbf{s}}^{\prime \prime}$ and $Q_{\mathbf{s}}^{\prime}$ denote the gradient and Hessian of $Q_{\mathbf{s}}$, respectively. From (16), (17), and (18) we obtain

$$
\left(A W_{\mathbf{s}} A^{T}\right) h=-A W_{\mathbf{s}} r-\gamma(A \mathbf{s}+b) .
$$

For ease of notation let $C \equiv A W_{\mathbf{s}} A^{T}$ and $g \equiv-A W_{\mathbf{s}} r-\gamma(A \mathbf{s}+b)$. Furthermore, let $\mathcal{N}(C)$ denote the null space of $C$. If $C$ has full rank, then $h$ is the solution of (36). Otherwise, if the system of equations (36) is consistent, $h$ is taken to be the minimum norm solution. If the system is inconsistent, $h$ is taken to be the projection of $g$ on $\mathcal{N}(C)$. The motivation for these choices is discussed in [12]. The next iterate is found by a line search aiming for a zero of the directional derivative. This procedure is computationally cheap as a result of the piecewise linear nature of $G_{\gamma}^{\prime}$. It is shown in [12] that the iteration is finite; i.e., after a finite number of iterations we have $x+h \in \mathcal{C}_{\mathbf{s}(x)}^{\gamma}$. Therefore, $x+h$ is a minimizer of $G_{\gamma}$ as a result of (15), (16), and the convexity of $G_{\gamma}$. We summarize below the modified Newton algorithm.

$$
\begin{aligned}
& \text { repeat } \\
& \qquad \mathbf{s}=\mathbf{s}_{\gamma}(x)
\end{aligned}
$$

if (36) consistent then 


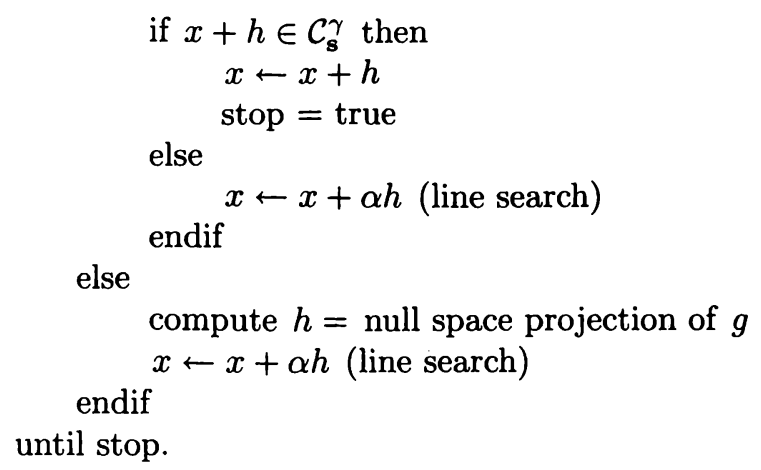

4.2. Checking optimality and reducing $\gamma$. Let $x_{\gamma}$ be a minimizer of $G_{\gamma}$ computed using the modified Newton algorithm of the previous section. Then either the continuation algorithm terminates or the modified Newton algorithm is restarted using a reduced value of $\gamma$. Let $\mathbf{s}=\mathbf{s}^{\gamma}\left(M_{\gamma}\right)$ and $d$ be a solution to (23). From the necessary condition (22) it follows that (23) is equivalent to

$$
\left(A W_{\mathbf{s}} A^{T}\right) d=-\frac{1}{\gamma} A W_{\mathbf{s}} r\left(x_{\gamma}\right) .
$$

Let $d$ solve (37), and let $r_{\gamma} \equiv r\left(x_{\gamma}\right)$. For $0 \leq \delta \leq \gamma$ we define

$$
x(\gamma-\delta)=x_{\gamma}+\delta d
$$

Then

$$
r(x(\gamma-\delta))=r_{\gamma}+\delta A^{T} d
$$

Notice that, following Lemma $3, x(\gamma-\delta)$ is a minimizer of $G_{\gamma-\delta}$ as long as $\mathbf{s}_{\gamma-\delta}(x(\gamma-$ $\delta))=\mathbf{s}$.

The stopping test is based on Theorem 4. It consists of checking the duality gap $\left(G(x(0))-c^{T} y_{\gamma}\right)$, where $y_{\gamma}$ is given in (32). If this is zero (within the roundoff tolerance) then the algorithm is stopped provided the residuals satisfy the following sign accordance:

$$
\begin{aligned}
& s_{\gamma i}=0 \Longrightarrow r_{i}(x(0))=0 \\
& s_{\gamma i} \neq 0 \Longrightarrow s_{\gamma i} r_{i}(x(0)) \geq 0 .
\end{aligned}
$$

Otherwise, we let $y(\gamma-\delta)=-\left(\frac{1}{\gamma-\delta} W_{\mathbf{s}} r(x(\gamma-\delta))+\mathbf{s}\right)$, and then we attempt to find the value $\delta^{*}$ of $\delta$ for which $\left|G(x(\gamma-\delta))-c^{T} y(\gamma-\delta)\right|$ attains its minimum. This is basically done by examining the points $\delta=\frac{k}{10} \gamma, k=1, \ldots, 10$, and choosing the best of these in the above sense provided that $\mathbf{s}_{\gamma-\delta^{*}}\left(x\left(\gamma-\delta^{*}\right)\right) \neq \mathbf{s}$. Then $x\left(\gamma-\delta^{*}\right)$ given by (38), with $r\left(x\left(\gamma-\delta^{*}\right)\right)$ given by (39), starts the Newton iteration using the new threshold value $\gamma-\delta^{*}$. The precise description of this procedure is given below.

$$
\begin{aligned}
& \Delta_{0}=G\left(x_{\gamma}\right)-c^{T} y(\gamma) \\
& k \leftarrow 0 \\
& \text { while not STOP do } \\
& \qquad k \leftarrow k+1 \\
& \quad \delta \leftarrow k * \gamma / 10
\end{aligned}
$$




$$
\begin{aligned}
& x \leftarrow x_{\gamma}+\delta d \\
& r \leftarrow r_{\gamma}+\delta A^{T} d \\
& y \leftarrow y(\gamma-\delta) \\
& \Delta_{k}=G(x)-c^{T} y
\end{aligned}
$$

end while

if $k \leq 2$ then $\delta \leftarrow 0.1 * \delta$

else $\delta \leftarrow(k-1) * \gamma / 10$

endif

$$
\begin{aligned}
& x \leftarrow x_{\gamma}+\delta d \\
& r \leftarrow r_{\gamma}+\delta A^{T} d \\
& \gamma \leftarrow \gamma-\delta
\end{aligned}
$$

STOP is a function that returns true when one of the following conditions hold:

$$
\Delta_{k}>\Delta_{k-1}, \quad \Delta_{k}<-0.1 \Delta_{0}
$$

provided that $\mathbf{s}_{\gamma-\delta}(x(\gamma-\delta))=\mathbf{s}$ or when

$$
k=9 .
$$

The first condition ensures that the procedure stops close to a minimum. The second condition guards against the case where the sign of the difference in the function values is reversed.

We have also experimented with alternative procedures for reducing $\gamma$. We used the total iteration count as the performance measure. The experiments showed that there is no significant difference among the alternative procedures in terms of performance. As a result, we adopted the above procedure since it has a sound justification as a result of Theorem 4 . We note that this procedure guarantees that unless the duality gap is closed, $\gamma$ is decreased by at least a factor of 0.9 after each unconstrained minimization. Hence, we have the following theorem.

THEOREM 5. The continuation algorithm described in $\S \S 4.1$ and 4.2 stops at an optimal solution $y^{*}$ of [NormLP] after a finite number of iterations.

Proof. As a result of the observation, $\gamma$ is reduced by a certain factor after each unconstrained minimization phase unless optimality is reached. Hence, using Theorem 1, $\gamma$ can only be decreased a finite number of times. Since the modified Newton algorithm is finite [12], the result follows.

5. Numerical results. In this section we report our numerical experience with a Fortran 77 implementation of the new algorithm, which does not exploit sparsity. We use two sets of test problems:

1. randomly generated full problems,

2. sparse problems from the Netlib collection [7].

We have three goals when we perform numerical experiments. The first goal is to examine the growth in solution time and iteration count of the new algorithm as the problem size is increased. The second goal is to test the numerical accuraey of the algorithm. We use randomly generated test problems to achieve these ends. The third goal is to test the viability of the algorithm in solving problems of practical interest. To accomplish this we choose a set of small, sparse test problems from the Netlib collection. Since these problems are very different in nature from the randomly generated problems this should give us insight into the potential of the new algorithm as a competitor to the well-known algorithms for LP. To this end, we compare our 
results with an LP subroutine E04MBF from the NAG subroutine library. E04MBF is based on LSSOL from Stanford Systems Optimization Library. It is a Fortran 77 package for constrained linear least squares problems, LP, and convex quadratic programming [9]. It does not exploit sparsity. Hence, it provides a fair comparison with our numerical results.

The major effort in the Newton algorithm of $\S 4.1$ is spent in solving the systems (36). We use the AAFAC package of [17] to perform this. The solution is obtained via an $L D L^{T}$ factorization of the matrix $C_{k}=A W_{\gamma}\left(x_{k}\right) A^{T}$, so $D$ and $L$ are computed directly from the active columns of $A$, i.e., without squaring the condition number as would be the case if $C_{k}$ was first computed. It is essential for the efficiency of the continuation algorithm that normally the $\gamma$-active set $\mathcal{A}_{\gamma}\left(x_{k}\right)$ differs relatively little from $\mathcal{A}_{\gamma}\left(x_{k-1}\right)$. This implies that the factorization of $C_{k}$ can be obtained by relatively few up- and downdates of the factorization of $C_{k-1}$. Therefore, the computational cost of a typical iteration step is $O\left(n^{2}\right)$. Occasionally, a refactorization is performed. This consists of the successive updating $L D L^{T} \leftarrow L D L^{T}+a_{j} a_{j}^{T}$ for all $j$ in the active set (starting with $L=I, D=0$ ). It is considered only when some columns of $A$ leave the active set, i.e., when downdating is involved. If many columns leave we may refactorize because it is cheaper. Otherwise, a refactorization is used when a downdating results in a rank decrease and there is an indication that rounding errors have marked influence. This part of the algorithm combines ideas from $[6,8]$. For details see $\S 2$ in [17]. The refactorization is an $O\left(n^{3}\right)$ process.

The solution of (23) is done via (37), and the matrix $A W_{\mathbf{s}} A^{T}$ is identical to the last $C_{k}$ used in the Newton iteration. Thus, the $L D L^{T}$ factorization is available. In order to enhance accuracy we use one step of iterative refinement when solving (37). For further details see [18]. We refer to the subroutine that implements the continuation algorithm as LPASL1. With the exception of some internal tolerance parameters (e.g., tolerances used for numerical checks for zero), LPASL1 does not allow any control over the execution of the algorithm. Hence, all the results reported in this study were obtained under identical algorithmic choices. We note that LPASL1 is available for distribution as a Fortran 77 subroutine. Details can be found in [16].

First we report computational results using randomly generated problems. Our random problems are generated using ideas similar to the generator detailed in $\S 4.1$ of [18]. All the problems are of the form [NormLP]. Half of the matrix $A$ in each problem was taken as the identity matrix to simulate the presence of slack columns. We solve ten problems of each size. The tests were performed on a DEC 3000 Alpha APX Model 500 running DEC OSF1 V1.2. The Fortran code was compiled using the -0 option of the DEC Fortran compiler. The ratio $\mu=m / n$ is kept constant at 2 . In all cases, the duality gap in LPASL1 was below $10^{-8}$. In Table 1, we report the average over ten problems of the following LPASL1 statistics: number of iterations, run time in CPU seconds, number of refactorizations, and number of $\gamma$-reductions. The column "iter" refers to the total number of iterations, i.e., the total number of solutions to (36) and (37), and "reduc" to the number of $\gamma$-reductions. The column "refac" refers to the total number of refactorizations in connection with the computations of the factors $L$ and $D$. In all cases, the cardinality of the set $\mathcal{A}_{0}\left(x_{0}\right)$ and the rank of the associated matrix $A W_{\mathbf{s}} A^{T}$ on termination are equal to $n$. The two columns under the heading LSSOL contain the run time statistics of LSSOL. The rightmost column displays the ratio run time of LSSOL/run time of LPASL1. In all cases, the optimal objective function values reported by LPASL1 and LSSOL agree to at least eight digits. All runs with LPASL1 and LSSOL were performed using default parameters; i.e., no fine 
TABLE 1

Solution statistics of LPASL1 and LSSOL using randomly generated problems (NA: not solved due to memory shortage).

\begin{tabular}{||c||c|c|c|c||c|c||c||}
\hline \hline \multicolumn{1}{|c||}{$m, n$} & \multicolumn{4}{c||}{ LPASL1 } & \multicolumn{2}{c||}{ LSSOL } & ratio \\
\hline \hline & iter & refac & reduc & CPU & iter & CPU & \\
\hline$(200,100)$ & 56.2 & 4 & 16.4 & 0.6 & 352.2 & 4.7 & 7.83 \\
$(320,160)$ & 74.1 & 4.4 & 22.2 & 2.6 & 713.5 & 21.8 & 8.38 \\
$(480,240)$ & 104.7 & 6 & 30.2 & 9.9 & 1300.7 & 91.3 & 9.22 \\
$(720,360)$ & 117.2 & 4.8 & 32.4 & 30.5 & 2312 & 360.4 & 11.81 \\
$(1080,540)$ & 165.2 & 5.4 & 44.3 & 116.5 & 4305.6 & 1470.9 & 12.62 \\
$(1620,810)$ & 217.4 & 8.5 & 51.7 & 469.4 & 8628.6 & 6501.7 & 13.85 \\
$(2430,1215)$ & 290.7 & 13.1 & 62.9 & 2648.7 & 16205.1 & 27777 & 10.48 \\
$(3644,1822)$ & 335 & 13.2 & 62.6 & 8327.3 & NA & NA & NA \\
\hline \hline
\end{tabular}

tuning of the codes was done for any test problem.

It was observed that over a set of ten problems of a given size, the performance statistics of LPASL1 display a larger variation around the mean than those of LSSOL. For instance, the minimum run time for problems of size $(2430,1215)$ was 1400 seconds while the maximum run time was 3569 seconds. For LSSOL, the two extreme values were 26441 seconds and 29113 seconds. However, we observe that LPASL1 performs increasingly better than LSSOL as the problem size is increased.

A least squares fit with the model $\beta \cdot n^{\alpha}$ shows that

$$
\begin{aligned}
& \operatorname{iter}_{L P A S L 1} \approx 3.0 \cdot n^{0.63}, \quad \operatorname{iter}_{L S S O L} \approx 0.3 \cdot n^{1.53}, \\
& \mathrm{CPU}_{L P A S L 1} \approx 1.2 \cdot 10^{-7} \cdot n^{3.31}, \quad \mathrm{CPU}_{L S S O L} \approx 4.7 \cdot 10^{-7} \cdot n^{3.48} .
\end{aligned}
$$

We observe that on both measures LSSOL exhibits a higher growth rate than LPASL1.

Finally we consider ten problems from the Netlib collection. We also used a test problem from a civil engineering application, referred to as PLATE. The characteristics of the problems are given in Table 2 .

TABLE 2

Characteristics of the sparse test problems.

\begin{tabular}{||l|c|c|c||}
\hline \hline Problem name & Constraints & Variables & Slack variables \\
\hline \hline afiro & 27 & 32 & 19 \\
sc50b & 50 & 48 & 30 \\
sc50a & 50 & 48 & 30 \\
sc105 & 105 & 103 & 60 \\
adlittle & 56 & 97 & 41 \\
scagr7 & 129 & 140 & 45 \\
stocfor1 & 117 & 111 & 54 \\
blend & 74 & 83 & 31 \\
sc205 & 205 & 203 & 114 \\
share2b & 96 & 79 & 83 \\
\hline plate & 61 & 73 & 60 \\
\hline \hline
\end{tabular}

All the problems we consider are of the form

$$
\begin{gathered}
\underset{y}{\operatorname{maximize}} c^{T} y \\
\text { subject to } \\
A y \leq b, \\
y \geq 0 .
\end{gathered}
$$


In order to apply the continuation algorithm to these problems, we prescribe a uniform bound $u$ to all the variables. Hence, we artificially transform the problem to the following equivalent problem:

$\begin{array}{cr}\underset{y}{\operatorname{maximize}} & c^{T} y \\ \text { subject to } & A y=b, \\ & 0 \leq y \leq U,\end{array}$

where $U=(u, \ldots, u)$. Then the problem can be transformed to the form [NormLP] by a simple linear transformation. No preprocessing was used prior to solving the problems. In Table 3, we show the solution statistics of LPASL1 and LSSOL. The tests were performed on an IBM PS/2 486. The Salford F77 compiler was used. The columns "iter," "reduc," and "refac" are as defined in Table 1. The columns " $\nu_{0}$ " and "rank" refer to the cardinality of the set $\mathcal{A}_{0}\left(x_{0}\right)$ and the rank of the associated matrix $A W_{\mathbf{s}} A^{T}$ on termination, respectively. Finally, we report the CPU time in seconds exclusive of input/output under "CPU." In all cases, the optimal objective function values reported by LPASL1 and LSSOL agree to at least eight digits. Both LPASL1 and LSSOL were run using default parameters.

TABLE 3

Solution statistics of LPASL1 and LSSOL on sparse problems.

\begin{tabular}{||l||c|c|c|c|c|c||c|c||}
\hline \multicolumn{1}{||c||}{ Problem name } & \multicolumn{7}{c||}{ LPASL1 } & \multicolumn{2}{c||}{ LSSOL } \\
\hline \hline & iter & refac & reduc & $\nu_{0}$ & rank & CPU & iter & CPU \\
\cline { 2 - 5 } & 21 & 4 & 4 & 28 & 27 & 0.49 & 19 & 0.60 \\
sc50b & 22 & 2 & 1 & 48 & 48 & 0.93 & 47 & 3.07 \\
sc50a & 32 & 3 & 3 & 47 & 47 & 1.64 & 40 & 2.47 \\
sc105 & 56 & 8 & 1 & 101 & 101 & 9.78 & 78 & 14.28 \\
adlittle & 89 & 18 & 15 & 62 & 56 & 8.13 & 149 & 14.56 \\
scagr7 & 76 & 7 & 10 & 129 & 129 & 19.06 & 126 & 58.07 \\
stocfor1 & 101 & 13 & 3 & 111 & 111 & 19.01 & 73 & 15.93 \\
blend & 60 & 8 & 3 & 73 & 72 & 7.25 & 85 & 12.96 \\
sc205 & 152 & 16 & 3 & 196 & 196 & 107.63 & 192 & 122.74 \\
share2b & 87 & 8 & 5 & 91 & 86 & 11.86 & 122 & 13.07 \\
\hline plate & 20 & 3 & 4 & 55 & 43 & 2.47 & 150 & 9.23 \\
\hline \hline
\end{tabular}

We observe that LPASL1 is faster than LSSOL on ten of the eleven problems. Furthermore, in the remaining problem, stocfor1, the performance figures of the two codes are very close. We expect to report results with all the Netlib problems in the future when we have an implementation that exploits sparsity.

Comparison with interior point and related methods. Our algorithm displays some similarities to interior point methods for LP and to the affine scaling methods of Coleman and $\mathrm{Li}$ in the main computational step. A common feature of all these algorithms is that their main computational step is the solution of a weighted least squares system of the form

$$
\left(A D A^{T}\right) p=f
$$

where $D$ is a diagonal positive definite matrix, and $p$ and $f$ are vectors of appropriate dimensions. Since the numerical values of the entries of $D$ change from one iteration to the next, a refactorization of the matrix $A D A^{T}$ is performed at each iteration of these methods. Since we do only occasional refactorizations of the matrix $A W A^{T}$ 
in our method, the cost of our average iteration, which consists of a series of upand downdates of the factors, is lower compared with that of the above-mentioned methods. Hence, the relative standing of our method with respect to these methods can only be established by a thorough computational comparison. To give the reader a feel for how the algorithms compare, we ran some of our randomly generated test problems using the CPLEX Barrier optimizer, which is a state-of-the-art implementation of a primal-dual interior point algorithm. The results are summarized in Table 4 below. These runs were made on a SUN 490 workstation.

TABLE 4

Solution statistics of LPASL1 and CPLEX on randomly generated problems.

\begin{tabular}{||c||c|c|c|c||c|c||}
\hline \hline$(m, n)$ & \multicolumn{4}{c||}{ LPASL1 } & \multicolumn{2}{c||}{ CPLEX barrier } \\
\hline \hline & iter & refac & reduc & CPU & iter & CPU \\
\cline { 2 - 7 } 200,100 & 43 & 8 & 5 & 19.95 & 13 & 16.95 \\
320,160 & 51 & 6 & 8 & 65.83 & 14 & 67.84 \\
400,200 & 58 & 4 & 12 & 104.90 & 15 & 140.39 \\
600,300 & 68 & 5 & 13 & 356.73 & 15 & 451.79 \\
\hline \hline
\end{tabular}

We notice that LPASL1 statistics in Table 4 differ from the results displayed in Table 1 for similar size problems. The explanation of this is the following. To carry out the tests reported in Table 4 we generated random problems where all columns were fully dense in contrast to our problems in Table 1 , where half of the matrix $A$ was taken as the identity matrix to simulate slack columns. The reason for this change is to force CPLEX Barrier to use dense linear algebra. Interestingly, we observed that LPASL1 consistently took fewer iterations and reductions on problems of the type used in Table 4 compared with its performance reported in Table 1. Computing times in Tables 1 and 4 also differ due to the difference in the computing platforms used for the two experiments.

We also observe in Table 4 that the iteration number in the interior point method grows very slowly. The time performances of LPASL1 and CPLEX Barrier are very close on these test problems with a slight advantage of LPASL1 as the problems get larger. Given the preliminary nature of our numerical work on the new algorithm, these results are encouraging.

We expect similar behavior from Coleman and Li's algorithm since it is reported in their paper that the iteration count of their algorithm is almost constant as a function of the problem size. We are not able to do any comparisons since they only have a MATLAB ${ }^{\mathrm{TM}}$ code.

6. Summary. In this paper, we described a new finite continuation algorithm for LP based on linear $\ell_{1}$ optimization. The algorithm solves a sequence of smooth nonlinear problems that yield a solution to the LP in a finite number of iterations. We studied the structure of the solution set of the approximating problems and showed how primal and dual solutions can be obtained. We also developed an efficient and stable implementation of the algorithm for full matrices and tested it on both randomly generated problems and some problems from the Netlib collection. Although these results are of a preliminary nature, they indicate that the algorithm exhibits a promising performance on both kinds of problems. We compared our results with a simplex LP routine from the Stanford Systems Optimization Library that is available as a subroutine in the NAG library, and with the CPLEX Barrier Optimizer. The comparison showed that the new algorithm is substantially faster than the NAG routine on randomly generated problems with full matrices. It is also competitive on the 
Netlib problems tried. The comparison with CPLEX also gave encouraging results. The following issues remain for further studies:

- "hot" starts,

- preprocessing and automatic choice of an upper bound for problems with only nonnegativity restrictions on the variables,

- exploiting sparsity.

\section{REFERENCES}

[1] R. H. BARTELS (1980), A penalty linear programming method using reduced-gradient basisexchange techniques, Linear Algebra Appl., 29, pp. 17-32.

[2] B. CHEN AND P.T. HARKER (1993), A non-interior continuation algorithm for linear and quadratic programming, SIAM J. Optim., 3, pp. 503-515.

[3] T. Coleman AND Y. LI (1990), A globally and quadratically convergent affine scaling algorithm for the linear programming problem with bounded variables, in Large Scale Numerical Optimization, T. Coleman and Y. Li, eds., Society for Industrial and Applied Mathematics, Philadelphia, PA, pp. 49-57.

[4] - (1992), A globally and quadratically convergent affine scaling algorithm for $\ell_{1}$ problems, Math. Programming, 56, pp. 189-222.

[5] A. R. ConN (1976), Linear programming via a nondifferentiable penalty function, SIAM J. Numer. Anal., 13, pp. 145-154.

[6] R. Fletcher AND M. J. D. Powell (1974), On the modification of $L D L^{T}$ factorizations, Math. Comp., 28, pp. 1067-1087.

[7] D. M. GAY (1985), Electronic mail distribution of linear programming test problems, Math. Prog. Soc. COAL Newsletter, 13, pp. 10-12.

[8] W. M. Gentleman (1973), Least squares computation by Givens transformations without square roots, J. Inst. Math. Appl., 12, pp. 329-336.

[9] P. E. Gill, S. Hammarling, W. Murray, M. A. Saunders, and M. H. Wright (1986), User's Guide for LSSOL (Version 1.0): A Fortran Package for Constrained Linear Least Squares and Convex Quadratic Programming, Report SOL 86-1, Department of Operations Research, Stanford University, Stanford, CA.

[10] P. HUBER (1981), Robust Statistics, John Wiley, New York.

[11] I. LuSTIG, R. MARSTEN, AND D. ShANNO (1991), Computational experience with a primal-dual interior point method for linear programming, Linear Algebra Appl., 152, pp. 191-222.

[12] K. MADSEN AND H.B. NiELSEN (1990), Finite algorithms for robust linear regression, BIT, 30, pp. 682-699.

[13] - (1993), A finite smoothing algorithm for linear $\ell_{1}$ estimation, SIAM J. Optim., 3, pp. 223-235.

[14] K. Madsen, H. B. Nielsen, And M. C. Pinar (1992), Linear, Quadratic and Minimax Programming Using $\ell_{1}$ Optimization, Report NI-92-11, Institute of Mathematical Modelling, Numerical Analysis Group, Technical University of Denmark, Lyngby, Denmark.

[15] - (1994), New characterizations of $\ell_{1}$ solutions to overdetermined linear systems, Oper. Res. Lett., 16, 3, pp. 159-166.

[16] (1994), User's Guide to LPASL1: A Fortran 77 Subroutine, Based on a Continuation Algorithm, for Dense Linear Programming, Report IMM-94-01, Institute of Mathematical Modelling, Numerical Analysis Group, Technical University of Denmark, Lyngby, Denmark.

[17] H. B. NiELSEN (1990), AAFAC: A Package of Fortran 77 Subprograms for Solving $A^{T} A x=$ $c$, Report NI-90-11, Institute of Mathematical Modelling, Numerical Analysis Group, Technical University of Denmark, Lyngby, Denmark.

[18] - (1991), Implementation of a Finite Algorithm for Linear $\ell_{1}$ Estimation, Report NI-9101, Institute of Mathematical Modelling, Numerical Analysis Group, Technical University of Denmark, Lyngby, Denmark.

[19] M. C.. PINAR AND S. A. Zenios (1994), On smoothing exact penalty functions for convex constrained optimization, SIAM J. Optim., 4, pp. 486-511.

[20] T. ROCKAFELlaR (1990), Computational schemes for large-scale problems in extended linearquadratic programming, Math. Programming, 48, pp. 447-474.

[21] J. Sun (1992), On the structure of convex piecewise quadratic functions, J. Optim. Theory Appl., 72, pp. 499-510. 